\title{
Potentials of Melastoma malabathricum Linn. Flower and Fruit Extracts as Antimicrobial Infusions
}

\author{
Siti Nurhadis Che Omar ${ }^{1}$, Janna Ong Abdullah ${ }^{1 *}$, Khairul Anuar Khairoji ${ }^{1}$, Sieo Chin Chin ${ }^{2}$, \\ Muhajir Hamid ${ }^{\mathbf{1}}$ \\ ${ }^{1}$ Department of Microbiology, Faculty of Biotechnology and Biomolecular Sciences, Universiti Putra Malaysia, Serdang, Malaysia; \\ ${ }^{2}$ Institute of Bioscience, Universiti Putra Malaysia, Serdang, Malaysia. \\ Email: janna@biotech.upm.edu.my
}

Received June 21 ${ }^{\text {st }}$, 2012; revised July $18^{\text {th }}, 2012$; accepted July $25^{\text {th }}, 2012$

\begin{abstract}
Melastoma malabathricum Linn. is a shrub that belongs to the family Melastomataceae and a common herbal plant used in folk medicines to treat inflamed wounds. This study was carried out with the aim to evaluate the inhibitory activities of different concentrations of the M. malabathricum Linn. flower and fruit crude extracts against a variety of microorganisms. The inhibitory effects of both extracts were tested against the microorganisms using the disc diffusion method. The lowest concentrations of the extracts producing inhibition zones against the test microorganisms were used to determine their Minimum Inhibitory Concentrations (MICs) and Minimum Microbicidal Concentrations (MMCs). Both crude extracts showed strong inhibitory activities against Gram-positive bacteria. The range of MIC values for the crude flower and fruit extracts on all the bacteria tested were 12.5 to $100.0 \mathrm{mg} / \mathrm{ml}$. Overall, Gram-positive bacteria were more susceptible to the crude extracts compared to Gram-negative species, potentiating a possible use of the extracts to inhibit or kill potential pathogens.
\end{abstract}

Keywords: Melastoma malabathricum Linn.; Flower; Fruit; Disc Diffusion Method; Minimal Inhibitory Concentration; Minimal Microbicidal Concentration

\section{Introduction}

In the present era, modern medicine develops very quickly and makes major contributions to worldwide diseases control. Despite the rapid growth in knowledge and techniques in modern medicine, the populations of developed and developing countries also show an increase interest in traditional medicines. According to the World Health Organization [1], the definition of traditional medicine may be summarized as the sum total of all the knowledge and practices, whether explicable or not, used in the diagnosis, prevention and elimination of physical, mental or social imbalances, and relying exclusively on practical experiences and observations handed down from generation to generation, whether verbally or in writing. Today, almost $80 \%$ of the Asian population relies on traditional medicine as a part of their health care regime.

Melastoma malabathricum Linn. is a shrub that belongs to the family Melastomataceae. It can be found abundantly in Malaysia. It comes with beautiful pink or purple flowers. The flower consists of five narrow reddish sepals and five purplish petals. The fruits are en-

${ }^{*}$ Corresponding author. capsulated and contain many non-endospermous seeds [2] with small embryos inside purplish pulps. M. malabathricum Linn. is rich with flavonoid compounds [3,4]. In folklore remedies, different parts of the plants are used for the treatment of various human aliments. The leaf, root and flower crude extracts of M. malabathricum Linn. had been used to treat toothache, wounds, diarrhea, antiinfection, scar prevention, and post-partum recovery [5]. Numerous pharmacological studies and clinical practices have reported that various parts of M. malabathricum Linn. plant possess biological functions such as antioxidant and anti-cancer [3], antiviral [6], anti-inflammatory, antinociceptive and anti-pyretic [7], and antiulcerogenic [8].

In this study we employed thirty-two microbial species to test the possible antimicrobial activity of M. malabathricum Linn. crude fruit and flower extracts. These species include the Gram-positive and Gram-negative bacteria and fungi, which are food spoilers but normal human body flora. Most of these species cause serious cases of food poisoning and hence, there is a need for the development of preservatives to eliminate their growth. However, research on antibacterial properties of $M$. $m a-$ labathricum Linn. is still scarce; only a few scientific 
articles reported some of these activities [9]. Looking at the myriad uses of $M$. malabathricum Linn. in folk medicine, it is therefore worthwhile to scientifically evaluate the antimicrobial activity of the crude flower and fruit extracts of M. malabathricum Linn. against the groups of microorganisms earlier mentioned.

\section{Materials and Methods}

\subsection{Plant Materials}

The flowers and fruits of Melastoma malabathricum L. were collected along the road sides in Universiti Putra Malaysia, Serdang, Selangor, Malaysia. Authentication of the plant was done at the Institute of Bioscience, Universiti Putra Malaysia, where the voucher specimen was conserved under the reference number SK1517/07.

\subsection{Extraction}

About $100 \mathrm{~g}$ of each fresh petals and fruits was extracted separately overnight with $1000 \mathrm{ml}$ of methanol (Merck, Darmstadt, Germany), at $25^{\circ} \mathrm{C} \pm 1^{\circ} \mathrm{C}$. The extracts were ducked through Whatman No. 1 filter paper (Whatman, Maidstone, England), and then vacuum dried in a rotary evaporator (Buchi, Flawil, Switzerland) at $37^{\circ} \mathrm{C} \pm 1^{\circ} \mathrm{C}$.

\subsection{Microbial Strains and Culture Conditions}

The test microorganisms used in this study are as listed in Table 1. ATCC strains were bought from the American Type Culture Collection (USA) and other strains of human origin were obtained from the Institute of Medical Research (IMR), Malaysia.

\subsection{Antimicrobial Assays}

\subsubsection{Preparation of Extract Test Samples}

Vacuum dried crude flower and fruit extracts of the following amounts: $600 \mathrm{mg}, 500 \mathrm{mg}, 400 \mathrm{mg}, 300 \mathrm{mg}, 200$ $\mathrm{mg}, 100 \mathrm{mg}$ were dissolved respectively in $1 \mathrm{ml}$ of water. Then, all the dissolved extracts were filtered through a $0.22 \mu \mathrm{m}$ membrane. A total of $10 \mu \mathrm{l}$ of each sample extracts was loaded onto a sterile $5 \mathrm{~mm}$ diameter paper discs. Sterilized water was used as a negative control. All impregnated discs were allowed to dry overnight at room temperature in a laminar flow hood. The commercial Tetracycline 30 (Oxoid, Hampshire, England) and Nystatin 100 (Oxoid, Hampshire, England) discs were used as positive controls for bacteria and fungi, respectively.

\subsubsection{Disc Diffusion Assay}

The antimicrobial activity was measured using the disc diffusion method [10]. All bacterial were grown for 18 $24 \mathrm{~h}$ at $37^{\circ} \mathrm{C}$ in Nutrient broth (Merck, Darmstadt, Germany) and the cultures were adjusted to match 0.5
McFarland standard. The fungi were cultured for 24 - 48 $\mathrm{h}$ in Potato Dextrose broth (BD Difco, Maryland, USA) at $30^{\circ} \mathrm{C}-37^{\circ} \mathrm{C}$. Petri dishes containing Nutrient agar (Merck, Darmstadt, Germany) and Potato Dextrose agar (BD Difco, Maryland, USA) were each swabbed with $100 \mu \mathrm{l}$ of the microbial suspensions (contained approximately $10^{6}$ to $10^{7} \mathrm{cfu} / \mathrm{ml}$ ). Sterile $5 \mathrm{~mm}$ diameter paper discs impregnated with $10 \mu$ leach of $100,200,300,400$, 500 and $600 \mathrm{mg} / \mathrm{ml}$ extracts were placed on the agar surface. A disc impregnated with water was used as a negative control. All the plates were incubated at $37^{\circ} \mathrm{C}$ for 24 $\mathrm{h}$ for the bacteria and $30^{\circ} \mathrm{C}-37^{\circ} \mathrm{C}$ for $24-48 \mathrm{~h}$ for the fungi. All tests were performed in triplicates and repeated three times.

\subsubsection{Macrodilution Method for Minimum Inhibitory Concentration (MIC) Determination}

The Minimum Inhibitory Concentration (MIC) was determined only for microbial species which showed growth inhibition zones in the disc diffusion method described above. The inoculum was prepared from a 18 $24 \mathrm{~h}$ broth culture using the disc diffusion method described above. A 18 - $24 \mathrm{~h}$ broth culture of each respecttive microbial species, adjusted to 0.5 McFarland standard, was used as the inoculum in this experiment. Approximately $100 \mathrm{mg}$ each of the crude flower and fruit extracts were dissolved in Nutrient broth separately in a test tube to a final concentration of $100 \mathrm{mg} / \mathrm{ml}$. A two folds dilution was prepared to give final concentrations of $0.39,0.78,1.56,3.12,6.25,12.5,25.0,50.0$ and 100.0 $\mathrm{mg} / \mathrm{ml}$ of the crude flower and fruit extracts. Then, 500 $\mu \mathrm{l}$ (contained approximately $10^{6}$ to $10^{7} \mathrm{cfu} / \mathrm{ml}$ ) of bacterial inoculum was added into each corresponding tube. Crude flower and fruit extracts without bacterial inoculum were used as negative controls. Tetracycline (Oxoid, Hampshire, England) was used as a positive control.

Culture growth was determined macroscopically and recorded after $18-24 \mathrm{~h}$ incubation at $37^{\circ} \mathrm{C}$. The MIC was determined as the lowest concentration corresponding to the test tube presenting no turbidity changed after incubation. All tests were performed in triplicates and repeated three times.

\subsubsection{Minimum Microbicidal Concentration (MMC) Determination}

For the determination of the Minimum Microbicidal Concentration (MMCs), $100 \mu \mathrm{l}$ of the content from each tested tube in the MIC assay that showed no turbidity changed was used to spread onto fresh Nutrient agar. The plates were further incubated for $18-24 \mathrm{~h}$ at $37^{\circ} \mathrm{C}$. The lowest concentration that yielded no growth, at which $99.9 \%$ of the bacterial was killed, was determined as the MMC [11]. All tests were performed in triplicates and repeated three times. 
Table 1. Microbial species, their cultivation and assays media used in this study.

\begin{tabular}{|c|c|c|c|}
\hline Microbial species & Strain & Cultivation medium & Assay medium \\
\hline Alcaligenes faecalis & IMR A111 & Nutrient agar & Nutrient agar \\
\hline Escherichia coli & IMR E113 & Nutrient agar & Nutrient agar \\
\hline Enterobacter aerogenes & IMR E153 & Nutrient agar & Nutrient agar \\
\hline Enterobacter aerogenes & ATCC 13048 & Nutrient agar & Nutrient agar \\
\hline Proteus mirabilis & IMR P184 & Nutrient agar & Nutrient agar \\
\hline Proteus vulgaris & IMR P147 & Nutrient agar & Nutrient agar \\
\hline Proteus vulgaris & ATCC 8427 & Nutrient agar & Nutrient agar \\
\hline Klebsiella pneumonia & IMR K36 & Nutrient agar & Nutrient agar \\
\hline Klebsiella pneumonia & ATCC 33495 & Nutrient agar & Nutrient agar \\
\hline Salmonella typhimurium & IMRS1205 & Nutrient agar & Nutrient agar \\
\hline Salmonella typhi & IMR S346 & Nutrient agar & Nutrient agar \\
\hline Shigella dysenteriae & IMR S340 & Nutrient agar & Nutrient agar \\
\hline Shigella sonnei & ATCC 2593 & Nutrient agar & Nutrient agar \\
\hline Serratia marcescens & IMR S913 & Nutrient agar & Nutrient agar \\
\hline Pseudomonas aeruginosa & IMR P187 & Nutrient agar & Nutrient agar \\
\hline Staphylococcus aureus & IMR S244 & Nutrient agar & Nutrient agar \\
\hline Staphylococcus aureus & IMR S942 & Nutrient agar & Nutrient agar \\
\hline Staphylococcus epidermidis & IMR S168 & Nutrient agar & Nutrient agar \\
\hline Bacillus subtilis & IMR B145 & Nutrient agar & Nutrient agar \\
\hline Bacillus cereus & IMR B43 & Nutrient agar & Nutrient agar \\
\hline Streptococcus pyogenes & IMR S1269 & Blood agar & Nutrient agar \\
\hline Streptococcus pyogenes & ATCC 19615 & Blood agar & Nutrient agar \\
\hline Listeria monocytogenes & IMR L55 & Nutrient agar & Nutrient agar \\
\hline Enterococcus faecalis & IMR E227 & Nutrient agar & Nutrient agar \\
\hline Enterococcus faecalis & ATCC 29212 & Nutrient agar & Nutrient agar \\
\hline Micrococcus luteus & IMR M174 & Nutrient agar & Nutrient agar \\
\hline Micrococcus luteus & ATCC 10240 & Nutrient agar & Nutrient agar \\
\hline Candida albicans & IMR C451 & Potato dextrose agar & Potato dextrose agar \\
\hline Candida albicans & ATCC 10231 & Potato dextrose agar & Potato dextrose agar \\
\hline Saccharomyces cerevisiae & IMR S1224 & Potato dextrose agar & Potato dextrose agar \\
\hline
\end{tabular}

\subsection{Data Analysis}

All the experiments were repeated three times. The statistical data such as means and standard deviations was calculated using ANOVA (SPSS software for windows, version 17.0). The significance of the differences was determined by Tukey's test at $P<0.05$ [12].

\section{Results and Discussion}

The average yield of the crude flower extract obtained from a starting $100 \mathrm{~g}$ of fresh petals was $6.0 \mathrm{~g}$. While, the average yield of the crude fruit extract obtained was $9.6 \mathrm{~g}$ from $100 \mathrm{~g}$ of fresh fruits.

Table 2 shows the inhibition zones of Melastoma malabathricum Linn. extracts against Gram-negative bacte- ria at $100-600 \mathrm{mg} / \mathrm{ml}$. The results showed that $S$. sonnei ATCC 2593 was the most sensitive candidate towards the crude flower extract, with a minimal inhibition zone of $13.2 \pm 0.2 \mathrm{~mm}$. While P. mirabilis IMR P184 and $P$. vulgaris IMR P147 exhibited the highest sensitivity towards the crude fruit extract with a $8.0 \pm 0.0 \mathrm{~mm}$ inhibittion zone size. E. coli IMR E113, E. aerogenes IMR E153, E. aerogenes ATCC 13048, K. pneumoniae IMR K36, K. pneumoniae ATCC 33495, S. typhi IMR S346 and S. typhimurium IMR S1205 were not inhibited by both extracts at all the concentrations tested.

Overall, the results showed that the flower and fruit extracts were more effective against the Gram-positive bacteria tested (Table 3). The highest inhibition was attributed by the flower extract against $M$. luteus ATCC 
Table 2. Inhibition zones (mm) of Melastoma malabathricum Linn. extracts against Gram-negative bacteria.

\begin{tabular}{|c|c|c|c|c|c|c|c|c|c|c|c|c|c|}
\hline \multirow{3}{*}{$\begin{array}{l}\text { Gram-negative } \\
\text { bacteria }\end{array}$} & \multicolumn{12}{|c|}{ Inhibition zone (mm) } & \multirow[b]{3}{*}{ Antibiotics $^{\mathrm{A}}$} \\
\hline & \multicolumn{6}{|c|}{ Flower extract (mg/ml) [extract yield (\%) in solvent] } & \multicolumn{6}{|c|}{ Fruit extract (mg/ml) [extract yield (\%) in solvent] } & \\
\hline & 100 & 200 & 300 & 400 & 500 & 600 & 100 & 200 & 300 & 400 & 500 & 600 & \\
\hline $\begin{array}{l}\text { A. faecalis } \\
\text { IMR A111 }\end{array}$ & $6.7 \pm 0.2^{\mathrm{a}}$ & $10.2 \pm 0.2^{\mathrm{b}}$ & $\begin{array}{c}11.2 \pm \\
0.7^{\mathrm{b}, \mathrm{c}}\end{array}$ & $\begin{array}{c}12.0 \pm \\
0.5^{\mathrm{c}}\end{array}$ & $\begin{array}{c}12.5 \pm \\
0.5^{\mathrm{c}}\end{array}$ & $\begin{array}{c}14.3 \pm \\
1.1^{\mathrm{d}}\end{array}$ & $\begin{array}{c}7.5 \pm \\
0.0^{\mathrm{a}}\end{array}$ & $\begin{array}{l}8.7 \pm \\
0.2^{\mathrm{a}, \mathrm{b}}\end{array}$ & $\begin{array}{c}10.7 \pm \\
0.2^{\mathrm{b}}\end{array}$ & $\begin{array}{c}11.5 \pm \\
0.5^{\mathrm{b}, \mathrm{c}}\end{array}$ & $\begin{array}{c}14.0 \pm \\
1.0^{\mathrm{c}, \mathrm{d}}\end{array}$ & $\begin{array}{c}14.5 \pm \\
0.5^{\mathrm{d}}\end{array}$ & $38.6 \pm 1.1$ \\
\hline $\begin{array}{l}\text { E. coli IMR } \\
\text { E113 }\end{array}$ & - & - & - & - & - & - & - & - & - & - & - & - & $8.6 \pm 0.2$ \\
\hline $\begin{array}{l}\text { E. aerogenes } \\
\text { ATCC } 13048\end{array}$ & - & - & - & - & - & - & - & - & - & - & - & - & $20.0 \pm 0.0$ \\
\hline $\begin{array}{l}\text { E. aerogenes } \\
\text { IMR E153 }\end{array}$ & - & - & - & - & - & - & - & - & - & - & - & - & $18.1 \pm 0.2$ \\
\hline $\begin{array}{l}\text { K. pneumoniae } \\
\text { ATCC } 33495\end{array}$ & - & - & - & - & - & - & - & - & - & - & - & - & $25.0 \pm 0.0$ \\
\hline $\begin{array}{l}\text { K. pneumoniae } \\
\text { IMR K } 36\end{array}$ & - & - & - & - & - & - & - & - & - & - & - & - & $23.6 \pm 2.0$ \\
\hline $\begin{array}{l}\text { P. mirabilis } \\
\text { IMR P184 }\end{array}$ & $11.8 \pm 0.7^{\mathrm{a}}$ & $\begin{array}{c}12.8 \pm \\
0.7^{\mathrm{a}, \mathrm{b}}\end{array}$ & $\begin{array}{c}13.5 \pm \\
0.5^{\mathrm{b}, \mathrm{c}}\end{array}$ & $\begin{array}{c}14.2 \pm \\
0.7^{\mathrm{c}, \mathrm{d}}\end{array}$ & $\begin{array}{c}14.8 \pm \\
0.7^{\mathrm{d}, \mathrm{e}}\end{array}$ & $\begin{array}{c}15.7 \pm \\
0.5^{\mathrm{e}}\end{array}$ & $\begin{array}{c}8.0 \pm \\
0.0^{\mathrm{a}}\end{array}$ & $\begin{array}{c}8.3 \pm \\
0.5^{\mathrm{a}}\end{array}$ & $\begin{array}{c}10.0 \pm \\
1.0^{\mathrm{a}, \mathrm{b}}\end{array}$ & $\begin{array}{c}11.2 \pm \\
1.0^{\mathrm{b}, \mathrm{c}}\end{array}$ & $\begin{array}{c}12.3 \pm \\
0.2^{\mathrm{c}}\end{array}$ & $\begin{array}{c}13.2 \pm \\
0.2^{c}\end{array}$ & $8.0 \pm 0.0$ \\
\hline $\begin{array}{l}\text { P. vulgaris } \\
\text { IMR P147 }\end{array}$ & $7.8 \pm 0.2^{\mathrm{a}}$ & $\begin{array}{l}10.7 \pm \\
0.2^{\mathrm{b}}\end{array}$ & $12.3 \pm 0.2^{c}$ & $\begin{array}{c}13.3 \pm \\
0.2^{\mathrm{d}}\end{array}$ & $\begin{array}{c}13.8 \pm \\
0.2^{\mathrm{d}}\end{array}$ & $\begin{array}{l}15.3 \pm \\
0.2^{\mathrm{e}}\end{array}$ & $\begin{array}{c}8.0 \pm \\
0.2^{\mathrm{a}}\end{array}$ & $\begin{array}{c}8.3 \pm \\
0.5^{\mathrm{a}}\end{array}$ & $\begin{array}{l}9.7 \pm \\
0.5^{\mathrm{a}, \mathrm{b}}\end{array}$ & $\begin{array}{l}10.0 \pm \\
0.0^{\mathrm{a}, \mathrm{b}, \mathrm{c}}\end{array}$ & $\begin{array}{c}11.3 \pm \\
0.5^{\mathrm{b}, \mathrm{c}}\end{array}$ & $\begin{array}{c}12.3 \pm \\
0.5^{\mathrm{c}}\end{array}$ & $23.6 \pm 0.5$ \\
\hline $\begin{array}{l}\text { P. vulgaris } \\
\text { ATCC } 8427\end{array}$ & - & $\begin{array}{c}10.8 \pm \\
0.2^{\mathrm{a}}\end{array}$ & $12.2 \pm 0.2^{\mathrm{a}}$ & $\begin{array}{l}13.2 \pm \\
0.2^{\mathrm{b}}\end{array}$ & $\begin{array}{c}14.8 \pm \\
0.5^{\mathrm{c}}\end{array}$ & $\begin{array}{c}18.2 \pm \\
1.1^{\mathrm{d}}\end{array}$ & - & $\begin{array}{c}9.0 \pm \\
0.5^{\mathrm{a}}\end{array}$ & $\begin{array}{l}10.3 \pm \\
0.2^{\mathrm{b}}\end{array}$ & $\begin{array}{l}11.8 \pm \\
0.2^{\mathrm{c}}\end{array}$ & $\begin{array}{c}13.2 \pm \\
0.2^{\mathrm{d}}\end{array}$ & $\begin{array}{c}14.5 \pm \\
0.5^{\mathrm{d}}\end{array}$ & $27.0 \pm 0.0$ \\
\hline $\begin{array}{l}\text { S. typhimurium } \\
\text { IMR S1205 }\end{array}$ & - & - & - & - & - & - & - & - & - & - & - & - & $28.0 \pm 0.0$ \\
\hline $\begin{array}{l}\text { S. typhi } \\
\text { IMR S346 }\end{array}$ & - & - & - & - & - & - & - & - & - & - & - & - & $28.6 \pm 1.1$ \\
\hline $\begin{array}{l}\text { S. dysenteriae } \\
\text { IMR S340 }\end{array}$ & $12.0 \pm 0.0^{\mathrm{a}}$ & $\begin{array}{c}13.5 \pm \\
0.5^{\mathrm{a}, \mathrm{b}}\end{array}$ & $\begin{array}{l}14.2 \pm \\
0.2^{\mathrm{b}}\end{array}$ & $\begin{array}{c}15.7 \pm \\
0.5^{\mathrm{c}}\end{array}$ & $\begin{array}{c}17.3^{ \pm} \\
0.5^{\mathrm{d}}\end{array}$ & $\begin{array}{c}18.0 \pm \\
0.0^{\mathrm{d}}\end{array}$ & - & $\begin{array}{c}9.7 \pm \\
0.5^{\mathrm{a}}\end{array}$ & $\begin{array}{c}11.0 \pm \\
0.0^{\mathrm{a}}\end{array}$ & $\begin{array}{c}11.7 \pm \\
0.5^{\mathrm{a}}\end{array}$ & $\begin{array}{c}12.3 \pm \\
0.5^{\mathrm{a}}\end{array}$ & $\begin{array}{c}13.3 \pm \\
1.1^{\mathrm{a}}\end{array}$ & $32.6 \pm 4.6$ \\
\hline $\begin{array}{l}\text { S. sonnei } \\
\text { ATCC } 2593\end{array}$ & $13.2 \pm 0.2^{\mathrm{a}}$ & $\begin{array}{c}14.2 \pm \\
0.2^{\mathrm{b}}\end{array}$ & $\begin{array}{c}15.0 \pm \\
0.0^{\mathrm{b}}\end{array}$ & $\begin{array}{c}15.8 \pm \\
0.2^{\mathrm{b}, \mathrm{c}}\end{array}$ & $\begin{array}{c}16.3 \pm \\
0.5^{\mathrm{c}, \mathrm{d}}\end{array}$ & $\begin{array}{c}17.5^{ \pm} \\
0.5^{\mathrm{d}}\end{array}$ & - & $\begin{array}{c}7.7 \pm \\
0.2^{\mathrm{a}}\end{array}$ & $\begin{array}{c}8.0 \pm \\
0.0^{\mathrm{a}}\end{array}$ & $\begin{array}{l}8.8 \pm \\
0.2^{\mathrm{b}}\end{array}$ & $\begin{array}{c}9.8 \pm \\
0.2^{\mathrm{c}}\end{array}$ & $\begin{array}{c}10.0 \pm \\
0.0^{\mathrm{c}}\end{array}$ & $18.0 \pm 0.0$ \\
\hline $\begin{array}{l}\text { P. aeruginosa } \\
\text { IMR P187 }\end{array}$ & $9.7 \pm 0.2^{\mathrm{a}}$ & $\begin{array}{c}12.3^{ \pm} \\
0.2^{\mathrm{b}}\end{array}$ & $\begin{array}{c}13.7 \pm \\
0.5^{\mathrm{b}, \mathrm{c}}\end{array}$ & $\begin{array}{c}15.3^{ \pm} \\
0.2^{\mathrm{d}}\end{array}$ & $\begin{array}{c}17.0 \pm \\
0.0^{\mathrm{e}}\end{array}$ & $\begin{array}{c}17.7 \pm \\
0.5^{\mathrm{e}}\end{array}$ & - & $\begin{array}{c}8.0 \pm \\
0.0^{\mathrm{a}}\end{array}$ & $\begin{array}{l}9.7 \pm \\
0.5^{\mathrm{a}, \mathrm{b}}\end{array}$ & $\begin{array}{c}10.2 \pm \\
0.7^{\mathrm{b}}\end{array}$ & $\begin{array}{r}11.0 \pm \\
1.0^{\mathrm{b}, \mathrm{c}}\end{array}$ & $\begin{array}{c}12.3 \pm \\
0.5^{\mathrm{c}}\end{array}$ & $14.3 \pm 1.1$ \\
\hline $\begin{array}{l}\text { S. marcescens } \\
\text { IMR S913 }\end{array}$ & - & $\begin{array}{l}8.8 \pm \\
0.2^{\mathrm{a}}\end{array}$ & $\begin{array}{l}9.8 \pm \\
0.2^{\mathrm{b}}\end{array}$ & $\begin{array}{c}10.7 \pm \\
0.2^{\mathrm{c}}\end{array}$ & $\begin{array}{c}11.3 \pm \\
0.2^{\mathrm{c}, \mathrm{d}}\end{array}$ & $\begin{array}{l}11.8 \pm \\
0.2^{\mathrm{d}}\end{array}$ & - & - & - & - & - & - & $19.6 \pm 0.5$ \\
\hline
\end{tabular}

-: No inhibition zone; ${ }^{\text {A }}$ Standard antibiotic (Tetracycline 30 for bacteria species); Negative control (100\% water) did not show inhibitory activity; Results are the means of inhibition zone values followed by standard deviations; Different letters within the same row indicate means at different concentrations for the same species are significantly different $(P<0.05)$.

Table 3. Inhibition zones (mm) of Melastoma malabathricum Linn. extracts against Gram-positive bacteria.

\begin{tabular}{|c|c|c|c|c|c|c|c|c|c|c|c|c|c|}
\hline \multirow{3}{*}{$\begin{array}{l}\text { Gram-positive } \\
\text { bacteria }\end{array}$} & \multicolumn{13}{|c|}{ Inhibition Zone (mm) } \\
\hline & \multicolumn{6}{|c|}{ Flower extract (mg/ml) [extract yield (\%) in solvent] } & \multicolumn{6}{|c|}{ Fruit extract (mg/ml) [extract yield (\%) in solvent] } & \multirow[b]{2}{*}{ Antibiotics $^{A}$} \\
\hline & 100 & 200 & 300 & 400 & 500 & 600 & 100 & 200 & 300 & 400 & 500 & 600 & \\
\hline $\begin{array}{l}\text { S. aureus IMR } \\
\text { S244 }\end{array}$ & $\begin{array}{c}9.7 \pm \\
0.5^{\mathrm{a}}\end{array}$ & $10.5 \pm 0.5^{\mathrm{a}, \mathrm{b}}$ & $\begin{array}{c}11.7 \pm \\
0.5^{\mathrm{b}, \mathrm{c}}\end{array}$ & $\begin{array}{c}12.8 \pm \\
0.2^{\mathrm{c}, \mathrm{d}}\end{array}$ & $\begin{array}{c}13.2 \pm \\
0.2^{\mathrm{d}}\end{array}$ & $\begin{array}{c}13.8 \pm \\
0.2^{\mathrm{d}}\end{array}$ & $\begin{array}{c}8.0 \pm \\
0.5^{\mathrm{a}}\end{array}$ & $\begin{array}{c}8.2 \pm \\
0.7^{\mathrm{a}}\end{array}$ & $\begin{array}{c}8.8 \pm \\
1.0^{\mathrm{a}}\end{array}$ & $\begin{array}{c}10.3 \pm \\
0.5^{\mathrm{a}, \mathrm{b}}\end{array}$ & $\begin{array}{c}11.2 \pm \\
0.7^{\mathrm{b}}\end{array}$ & $\begin{array}{c}12.0 \pm \\
1.0^{\mathrm{b}}\end{array}$ & $30.0 \pm 0.0$ \\
\hline $\begin{array}{l}\text { S. aureus IMR } \\
\text { S942 }\end{array}$ & $\begin{array}{c}7.0 \pm \\
0.0^{\mathrm{a}}\end{array}$ & $8.2 \pm 0.2^{\mathrm{a}, \mathrm{b}}$ & $\begin{array}{l}9.0 \pm \\
0.0^{\mathrm{b}, \mathrm{c}}\end{array}$ & $\begin{array}{l}9.8 \pm \\
0.2^{\mathrm{b}, \mathrm{c}}\end{array}$ & $\begin{array}{c}10.7 \pm \\
1.1^{\mathrm{c}, \mathrm{d}}\end{array}$ & $\begin{array}{c}12.0 \pm \\
1.0^{\mathrm{d}}\end{array}$ & $\begin{array}{c}7.0 \pm \\
0.2^{\mathrm{a}}\end{array}$ & $\begin{array}{c}8.2 \pm \\
0.2^{\mathrm{a}}\end{array}$ & $\begin{array}{l}9.0 \pm \\
0.0^{\mathrm{a}, \mathrm{b}}\end{array}$ & $\begin{array}{c}9.8 \pm \\
0.2^{\mathrm{b}}\end{array}$ & $\begin{array}{c}10.7 \pm \\
0.7^{\mathrm{b}, \mathrm{c}}\end{array}$ & $\begin{array}{c}12.0 \pm \\
1.0^{\mathrm{d}}\end{array}$ & $26.0 \pm 1.0$ \\
\hline $\begin{array}{l}\text { S. epidermidis } \\
\text { IMR S168 }\end{array}$ & $\begin{array}{c}10.3 \pm \\
0.2^{\mathrm{a}}\end{array}$ & $11.7 \pm 0.2^{\mathrm{b}}$ & $\begin{array}{c}12.3 \pm \\
0.2^{\mathrm{b}, \mathrm{c}}\end{array}$ & $\begin{array}{c}12.8 \pm \\
0.2^{\mathrm{c}, \mathrm{d}}\end{array}$ & $\begin{array}{c}13.3 \pm \\
0.2^{\mathrm{d}, \mathrm{e}}\end{array}$ & $\begin{array}{c}13.8 \pm \\
0.2^{\mathrm{e}}\end{array}$ & $\begin{array}{c}7.8 \pm \\
0.2^{\mathrm{a}}\end{array}$ & $\begin{array}{c}7.8 \pm \\
0.2^{\mathrm{a}}\end{array}$ & $\begin{array}{c}7.8 \pm \\
0.2^{\mathrm{a}}\end{array}$ & $\begin{array}{c}9.7 \pm \\
0.2^{\mathrm{b}}\end{array}$ & $\begin{array}{c}10.3 \pm \\
0.5^{\mathrm{b}, \mathrm{c}}\end{array}$ & $\begin{array}{c}11.3 \pm \\
0.5^{\mathrm{c}}\end{array}$ & $10.6 \pm 0.2$ \\
\hline $\begin{array}{l}\text { B. cereus IMR } \\
\text { B43 }\end{array}$ & $\begin{array}{c}6.2 \pm \\
0.2^{\mathrm{a}}\end{array}$ & $6.8 \pm 0.2^{\mathrm{b}}$ & $9.0 \pm 0.0^{c}$ & $\begin{array}{c}10.0 \pm \\
0.0^{\mathrm{d}}\end{array}$ & $\begin{array}{c}10.0 \pm \\
0.0^{\mathrm{d}}\end{array}$ & $\begin{array}{c}11.0 \pm \\
0.0^{\mathrm{e}}\end{array}$ & $\begin{array}{c}7.8 \pm \\
0.2^{\mathrm{a}}\end{array}$ & $\begin{array}{c}8.8 \pm \\
0.2^{\mathrm{b}}\end{array}$ & $\begin{array}{c}10.0 \pm \\
0.5^{c}\end{array}$ & $\begin{array}{c}10.5 \pm \\
0.5^{c}\end{array}$ & $\begin{array}{c}11.5 \pm \\
0.5^{\mathrm{d}}\end{array}$ & $\begin{array}{c}12.5 \pm \\
0.7^{\mathrm{d}}\end{array}$ & $30.0 \pm 0.0$ \\
\hline $\begin{array}{l}\text { B. subtilis IMR } \\
\text { B145 }\end{array}$ & $\begin{array}{c}9.2 \pm \\
0.2^{\mathrm{a}}\end{array}$ & $10.2 \pm 0.2^{\mathrm{b}}$ & $\begin{array}{c}10.8 \pm \\
0.2^{\mathrm{b}, \mathrm{c}}\end{array}$ & $\begin{array}{c}11.2 \pm \\
0.2^{\mathrm{c}, \mathrm{d}}\end{array}$ & $\begin{array}{c}11.8 \pm \\
0.2^{\mathrm{d}}\end{array}$ & $\begin{array}{c}12.0 \pm \\
0.5^{\mathrm{d}}\end{array}$ & $\begin{array}{c}6.5 \pm \\
0.0^{\mathrm{a}}\end{array}$ & $\begin{array}{c}6.5 \pm \\
0.0^{\mathrm{a}}\end{array}$ & $\begin{array}{l}8.8 \pm \\
0.2^{\mathrm{b}}\end{array}$ & $\begin{array}{c}10.3 \pm \\
0.5^{\mathrm{b}, \mathrm{c}}\end{array}$ & $\begin{array}{c}11.7 \pm \\
1.1^{\mathrm{c}, \mathrm{d}}\end{array}$ & $\begin{array}{c}12.8 \pm \\
0.7^{\mathrm{d}}\end{array}$ & $30.6 \pm 0.5$ \\
\hline $\begin{array}{l}\text { S. pyogenes } \\
\text { IMR S1269 }\end{array}$ & $\begin{array}{c}8.2 \pm \\
0.2^{\mathrm{a}}\end{array}$ & $9.2 \pm 0.2^{\mathrm{b}}$ & $\begin{array}{c}10.3 \pm \\
0.2^{\mathrm{c}}\end{array}$ & $\begin{array}{c}11.2 \pm \\
0.2^{\mathrm{d}}\end{array}$ & $\begin{array}{c}11.7 \pm \\
0.2^{\mathrm{d}, \mathrm{e}}\end{array}$ & $\begin{array}{c}12.3 \pm \\
0.2^{\mathrm{e}}\end{array}$ & - & $\begin{array}{c}7.0 \pm \\
0.0^{\mathrm{a}}\end{array}$ & $\begin{array}{l}7.5 \pm \\
0.5^{\mathrm{a}, \mathrm{b}}\end{array}$ & $\begin{array}{l}8.7 \pm \\
0.5^{\mathrm{b}, \mathrm{c}}\end{array}$ & $\begin{array}{l}9.8 \pm \\
0.5^{\mathrm{c}, \mathrm{d}}\end{array}$ & $\begin{array}{c}10.3 \pm \\
0.5^{\mathrm{d}}\end{array}$ & $26.6 \pm 0.5$ \\
\hline
\end{tabular}




\begin{tabular}{|c|c|c|c|c|c|c|c|c|c|c|c|c|c|}
\hline \multicolumn{14}{|l|}{ Continued } \\
\hline $\begin{array}{l}\text { S. pyogenes } \\
\text { ATCC } 19615\end{array}$ & $\begin{array}{c}8.3 \pm \\
0.2^{\mathrm{a}}\end{array}$ & $\begin{array}{c}9.8 \pm \\
0.2^{\mathrm{b}}\end{array}$ & $\begin{array}{c}12.5 \pm \\
0.5^{c}\end{array}$ & $\begin{array}{c}15.5 \pm \\
0.5^{\mathrm{d}}\end{array}$ & $\begin{array}{c}17.2 \pm \\
0.2^{\mathrm{e}}\end{array}$ & $\begin{array}{c}18.3 \pm \\
0.5^{\mathrm{f}}\end{array}$ & $\begin{array}{c}7.8 \pm \\
0.5^{\mathrm{a}}\end{array}$ & $\begin{array}{c}9.0 \pm \\
0.0^{\mathrm{b}}\end{array}$ & $\begin{array}{c}10.2 \pm \\
0.2^{\mathrm{b}, \mathrm{c}}\end{array}$ & $\begin{array}{c}11.8 \pm \\
0.2^{\mathrm{c}, \mathrm{d}}\end{array}$ & $\begin{array}{c}12.1 \pm \\
0.5^{\mathrm{d}, \mathrm{e}}\end{array}$ & $\begin{array}{c}12.4 \pm \\
0.5^{\mathrm{e}}\end{array}$ & $31.3 \pm 0.2$ \\
\hline $\begin{array}{l}\text { L. monocytogenes } \\
\text { IMR L55 }\end{array}$ & $\begin{array}{c}13.0 \pm \\
0.5^{\mathrm{a}}\end{array}$ & $\begin{array}{c}14.0 \pm \\
0.0^{\mathrm{a}}\end{array}$ & $\begin{array}{c}15.8 \pm \\
0.2^{\mathrm{b}}\end{array}$ & $\begin{array}{c}18.2 \pm \\
0.2^{\mathrm{c}}\end{array}$ & $\begin{array}{c}21.7 \pm \\
0.5^{\mathrm{d}}\end{array}$ & $\begin{array}{c}24.3 \pm \\
0.5^{\mathrm{e}}\end{array}$ & $\begin{array}{c}8.8 \pm \\
1.0^{\mathrm{a}}\end{array}$ & $\begin{array}{c}13.7 \pm \\
0.5^{\mathrm{b}}\end{array}$ & $\begin{array}{c}15.3 \pm \\
0.2^{\mathrm{b}, \mathrm{c}}\end{array}$ & $\begin{array}{l}15.7 \pm \\
0.5^{\mathrm{b}, \mathrm{c}, \mathrm{d}}\end{array}$ & $\begin{array}{c}16.7 \pm \\
0.5^{\mathrm{c}, \mathrm{d}}\end{array}$ & $\begin{array}{c}17.8 \pm \\
1.1^{\mathrm{d}}\end{array}$ & $29.3 \pm 0.5$ \\
\hline $\begin{array}{l}\text { E. faecalis IMR } \\
\text { E227 }\end{array}$ & - & - & $\begin{array}{c}9.7 \pm \\
0.5^{\mathrm{a}}\end{array}$ & $\begin{array}{c}10.2 \pm \\
0.7^{\mathrm{a}}\end{array}$ & $\begin{array}{c}10.7 \pm \\
0.7^{\mathrm{a}}\end{array}$ & $\begin{array}{c}11.2 \pm \\
0.7^{\mathrm{a}}\end{array}$ & - & $\begin{array}{c}6.8 \pm \\
0.2^{\mathrm{a}}\end{array}$ & $\begin{array}{l}7.7 \pm \\
0.2^{\mathrm{a}, \mathrm{b}}\end{array}$ & $\begin{array}{l}9.2 \pm \\
1.0^{\mathrm{a}, \mathrm{b}, \mathrm{c}}\end{array}$ & $\begin{array}{c}10.7 \pm \\
0.5^{\mathrm{b}, \mathrm{c}}\end{array}$ & $\begin{array}{c}11.7 \pm \\
0.5^{\mathrm{c}}\end{array}$ & $12.8 \pm 0.7$ \\
\hline $\begin{array}{l}\text { E. faecalis } \\
\text { ATCC } 19212\end{array}$ & - & $\begin{array}{c}8.0 \pm \\
0.5^{\mathrm{a}}\end{array}$ & $\begin{array}{c}9.2 \pm \\
0.2^{\mathrm{b}}\end{array}$ & $\begin{array}{c}10.3 \pm \\
0.5^{\mathrm{b}, \mathrm{c}}\end{array}$ & $\begin{array}{l}11.3 \pm \\
0.2^{\mathrm{c}, \mathrm{d}}\end{array}$ & $\begin{array}{c}11.0 \pm \\
0.2^{\mathrm{c}, \mathrm{d}}\end{array}$ & - & $\begin{array}{c}7.8 \pm \\
0.2^{\mathrm{a}}\end{array}$ & $\begin{array}{l}8.2 \pm \\
0.2^{\mathrm{a}, \mathrm{b}}\end{array}$ & $\begin{array}{c}9.3 \pm \\
0.5^{\mathrm{b}}\end{array}$ & $\begin{array}{c}10.2 \pm \\
0.2^{\mathrm{c}}\end{array}$ & $\begin{array}{c}10.8 \pm \\
0.2^{\mathrm{c}, \mathrm{d}}\end{array}$ & $23.0 \pm 0.5$ \\
\hline $\begin{array}{l}\text { M. luteus } \\
\text { ATCC } 10240\end{array}$ & $\begin{array}{c}26.7 \pm \\
0.5^{\mathrm{a}}\end{array}$ & $\begin{array}{c}30.3 \pm \\
0.5^{\mathrm{b}}\end{array}$ & $\begin{array}{c}31.7 \pm \\
0.5^{\mathrm{b}, \mathrm{c}}\end{array}$ & $\begin{array}{c}35.0 \pm \\
0.0^{\mathrm{d}}\end{array}$ & $\begin{array}{c}35.7 \pm \\
0.2^{\mathrm{d}}\end{array}$ & $\begin{array}{c}36.8 \pm \\
0.2^{\mathrm{e}}\end{array}$ & $\begin{array}{c}23.0 \pm \\
0.5^{\mathrm{a}}\end{array}$ & $\begin{array}{c}24.7 \pm \\
0.5^{\mathrm{a}, \mathrm{b}}\end{array}$ & $\begin{array}{c}25.7 \pm \\
0.5^{\mathrm{b}}\end{array}$ & $\begin{array}{c}28.0 \pm \\
0.5^{\mathrm{c}}\end{array}$ & $\begin{array}{c}29.7 \pm \\
0.2^{\mathrm{c}, \mathrm{d}}\end{array}$ & $\begin{array}{c}31.0 \pm \\
0.2^{\mathrm{d}}\end{array}$ & 66.330 .5 \\
\hline $\begin{array}{l}\text { M. luteus IMR } \\
\text { M174 }\end{array}$ & $\begin{array}{c}20.7 \pm \\
0.5^{\mathrm{a}}\end{array}$ & $\begin{array}{c}24.7 \pm \\
0.5^{\mathrm{b}}\end{array}$ & $\begin{array}{c}26.7 \pm \\
1.1^{\mathrm{b}, \mathrm{c}}\end{array}$ & $\begin{array}{c}28.3 \pm \\
0.5^{\mathrm{c}, \mathrm{d}}\end{array}$ & $\begin{array}{c}29.3 \pm \\
0.5^{\mathrm{c}, \mathrm{d}}\end{array}$ & $\begin{array}{l}31.3 \pm \\
2.0^{\mathrm{d}}\end{array}$ & $\begin{array}{c}31.0 \pm \\
0.5^{\mathrm{a}}\end{array}$ & $\begin{array}{c}36.0 \pm \\
0.5^{\mathrm{a}, \mathrm{b}}\end{array}$ & $\begin{array}{c}37.0 \pm \\
1.1^{\mathrm{b}}\end{array}$ & $\begin{array}{l}42.7 \pm \\
0.2^{\mathrm{c}}\end{array}$ & $\begin{array}{c}43.0 \pm \\
0.5^{\mathrm{c}, \mathrm{d}}\end{array}$ & $\begin{array}{c}46.7 \pm \\
2.0^{\mathrm{d}}\end{array}$ & $49.6 \pm 0.5$ \\
\hline
\end{tabular}

-: No inhibition zone; ${ }^{\text {A }}$ Standard antibiotic (Tetracycline 30 for bacteria species); Negative control (100\% water) did not show inhibitory activity; Results are the means of inhibition zone values followed by standard deviations; Different letters within the same row indicate means at different concentrations for the same species are significantly different $(P<0.05)$.

10240 with an inhibition zone of $26.7 \pm 0.5 \mathrm{~mm}$. Likewise, M. luteus IMR M174 was reported to be the most sensitive candidate towards the crude fruit extract with an inhibition zone of $31.0 \pm 0.5 \mathrm{~mm}$. These results validate the traditional medicine claim on M. malabathricum Linn. as having therapeutic potency to relief toothache and to treat wounds [5]. M. luteus is an opportunistic pathogen, and commonly found in human nasal cavity and on mammalian skin.

Both extracts did not exhibit any effects on the fungal species tested in this study (Table 4). This result is similar to a previous study by [13], who reported that grape seed extract containing pro-anthocyanidin compounds showed no antifungal activity. Similarly, Duraipandiyan and Ignacimutu [14] revealed that the methanol extracts of Cassia fistula flower showed no antifungal activity against $C$. albicans. All these findings could be attributed to the nature of the fungal's cell wall structure. Candida and Saccharomyces are types of yeast which possess polysaccharides, rigid and chemically unique cell walls containing chitin and glucan [15].

The antimicrobial activities of the crude flower and fruit extracts were further confirmed using the microdilution broth assay. The MIC and MMC values were summarized in Table 5. Both extracts exhibited antibacterial activity against the Gram-positive and -negative bacteria, with the MIC values ranging from 12.5 to $100.0 \mathrm{mg} / \mathrm{ml}$ for the flower extract and 6.3 to $100.0 \mathrm{mg} / \mathrm{ml}$ for the fruit extract. The lowest MIC value of $12.5 \mathrm{mg} / \mathrm{ml}$ was obtained using the crude flower extract on $B$. cereus IMR B43, S. pyogenes IMR S1269 and S. pyogenes ATCC 19615. For the crude fruit extract, the lowest MIC value of $12.5 \mathrm{mg} / \mathrm{ml}$ was obtained against L. monocytogenes IMR L55, S. aureus IMR S942, S. epidermis IMR S168, B. subtilis IMR B145 and S. pyogenes ATCC 19615. The average MMC values obtained varied from 50.0 to 100.0 $\mathrm{mg} / \mathrm{ml}$ for both extracts.

Overall, the results indicated that Gram-positive bacte- ria were more sensitive towards the crude flower and fruit extracts. Such observation is consistent with a previous study by Cushnie and Lamb [16], who reported that certain groups of flavonoid compounds exhibited greater inhibition effect on Gram-positive bacteria compared to Gram-negative bacteria. This was also consistent with previous studies on other plant species of $\mathrm{Me}$ lastomataceae [17-19]. The high sensitivity of Grampositive bacteria may be due to their cell wall and outer membrane structures [20]. Gram-negative bacteria possess an outer membrane and a unique periplasmic space not found in Gram-positive bacteria [21,22]. The resistance of Gram-negative bacteria towards antibacterial substances might also be related to the hydrophilic surface of their outer membrane, composing of lipopolysaccharide molecules, that present a barrier to the penetration of numerous antibiotic molecules and is also associated with the enzymes in the periplasmic space, which are capable of breaking down the molecules introduced from outside [23]. Gram-positive bacteria do not have such outer membrane and cell wall structures. Antibacterial substances can easily destroy the bacteria cell wall and cytoplasmic membrane resulting in a leakage of cytoplasmic materials extracellularly [24].

All concentrations of the extracts tested showed significant differences in the size of the inhibition zone produced (data not shown). This observation revealed that greater inhibition was observed with flower extract compared to fruit extract against all the bacteria tested. This might be due to the presence of different active compounds in the flower and fruit extracts. Susanti [3] reported that the ethyl acetate extract of M. malabathricum Linn. flower contained three different compounds such as kaempferol-3-O- $\beta$-D-glucoside, kaempferol and naringenin, while the ethyl acetate extract of the fruit contained betulinic acid. Other active compounds such as malvidin-3,5-diglucoside was found in the flowers while cyanidin-3-glucoside and cyanidin-3,5-diglucoside were 
Table 4. Inhibition zones (mm) of Melastoma malabathricum Linn. extracts against fungi.

\begin{tabular}{|c|c|c|c|c|c|c|c|c|c|c|c|c|c|}
\hline \multirow{3}{*}{ Fungi } & \multicolumn{12}{|c|}{ Inhibition zone (mm) } & \multirow[b]{3}{*}{ Antibiotics $^{\mathrm{A}}$} \\
\hline & \multicolumn{6}{|c|}{ Flower extract (mg/ml) [extract yield (\%) in solvent] } & \multicolumn{6}{|c|}{ Fruit extract (mg/ml) [extract yield (\%) in solvent] } & \\
\hline & 100 & 200 & 300 & 400 & 500 & 600 & 100 & 200 & 300 & 400 & 500 & 600 & \\
\hline C. albicans ATCC 10231 & - & - & - & - & - & - & - & - & - & - & - & - & $30.0 \pm 0.0$ \\
\hline C. albicans IMR C451 & - & - & - & - & - & - & - & - & - & - & - & - & $27.0 \pm 0.0$ \\
\hline S. cerevisiae IMR S1224 & - & - & - & - & - & - & - & - & - & - & - & - & $30.0 \pm 0.0$ \\
\hline
\end{tabular}

Table 5. MIC and MMC (mg/ml) values of Melastoma malabathricum Linn. extracts against selected microorganisms.

\begin{tabular}{|c|c|c|c|c|}
\hline \multirow{2}{*}{ Microbial strains } & \multicolumn{2}{|c|}{ Flower extract $(\mathrm{mg} / \mathrm{ml})$} & \multicolumn{2}{|c|}{ Fruit extract $(\mathrm{mg} / \mathrm{ml})$} \\
\hline & MIC & MMC & MIC & MMC \\
\hline \multicolumn{5}{|l|}{ Gram-negative bacteria } \\
\hline Alcaligenes faecalis IMR A111 & 100.0 & 100.0 & 50.0 & 100.0 \\
\hline Escherichia coli IMR E113 & N.D. & N.D. & N.D. & N.D. \\
\hline Enterobacter aerogenes ATCC 13048 & N.D. & N.D. & N.D. & N.D. \\
\hline Enterobacter aerogenes IMR E153 & N.D. & N.D. & N.D. & N.D. \\
\hline Klebsiella pneumoniae ATCC 33495 & N.D. & N.D. & N.D. & N.D. \\
\hline Klebsiella pneumoniae IMR K36 & N.D. & N.D. & N.D. & N.D. \\
\hline Proteus mirabilis IMR P184 & 50.0 & $>100.0$ & 25.0 & 100.0 \\
\hline Proteus vulgaris IMR P147 & 100.0 & 100.0 & 50.0 & 100.0 \\
\hline Proteus vulgaris ATCC 8427 & N.D. & N.D. & 100.0 & 100.0 \\
\hline Salmonella typhimurium IMR S1205 & N.D. & N.D. & N.D. & N.D. \\
\hline Salmonella typhi IMR S346 & N.D. & N.D. & N.D. & N.D. \\
\hline Shigella dysentriae IMR S340 & 12.5 & 100.0 & N.D. & N.D. \\
\hline Shigella sonnei ATCC 2593 & 12.5 & 100.0 & N.D. & N.D. \\
\hline Pseudomonas aeruginosa IMR P187 & 100.0 & 100.0 & N.D. & N.D. \\
\hline Serratia marcescens IMR S913 & N.D. & N.D. & N.D. & N.D. \\
\hline \multicolumn{5}{|l|}{ Gram -positive bacteria } \\
\hline Staphylococcus aureus IMR S244 & 100.0 & 100.0 & 100.0 & 100.0 \\
\hline Staphylococcus aureus IMR S942 & 100.0 & $>100.0$ & 12.5 & 100.0 \\
\hline Staphylococcus epidermidis IMR S168 & 50.0 & 50.0 & 12.5 & 100.0 \\
\hline Bacillus cereus IMR B43 & 12.5 & 50.0 & 25.0 & 100.0 \\
\hline Bacillus subtilis IMR B145 & 100.0 & 100.0 & 12.5. & 100.0 \\
\hline Streptococcus pyogenes IMR S1269 & 12.5 & 50.0 & N.D. & N.D. \\
\hline Streptococcus pyogenes ATCC 19615 & 12.5 & 50.0 & 12.5 & 50.0 \\
\hline Listeria monocytogenes IMR L55 & 12.5 & 100.0 & 12.5 & 100.0 \\
\hline Enterococcus faecalis IMR E227 & N.D. & N.D. & N.D. & N.D. \\
\hline Enteracoccus faecalis ATCC 19212 & N.D. & N.D. & N.D. & N.D. \\
\hline Micrococcus luteus ATCC 10240 & 100.0 & 100.0 & 100.0 & 100.0 \\
\hline Micrococcus luteus IMR M174 & 50.0 & 100.0 & 100.0 & 100.0 \\
\hline \multicolumn{5}{|l|}{ Fungi species } \\
\hline Candida albicans ATCC 10231 & N.D. & N.D. & N.D. & N.D. \\
\hline Candida albicans IMR C451 & N.D & N.D. & N.D & N.D. \\
\hline Saccharomyces cerevisiae IMR S1224 & N.D. & N.D. & N.D. & N.D. \\
\hline
\end{tabular}

Concentrations of extracts range from 0.39 to $100 \mathrm{mg} / \mathrm{ml}$; N.D.: No activity detected; Results are done in triplicates. 
found in the fruits [4]. These previous studies implied that the flower and fruit extracts of M. malabathricum Linn. contain different flavonoid compounds. According to Cushnie and Lamb [16], the different structural features of flavonoids may target different cellular components thus affecting different metabolic functions in a bacterial cell.

It is critical to note that an important factor that affects the size of the inhibition zone of antimicrobial activities using the disc diffusion method is the molecular weight of the bioactive compounds in the extract or antibiotics tested. Higher molecular weight antibiotics tend to have difficulty in diffusing into the agar medium, resulting in none or small inhibition zones. As was observed in a previous report that cranberry extract, containing lowmolecular-weight phenolic acids and flavanoids such as anthocyanins and flavonols, showed the greatest antibacterial properties [25]. But this does not mean the target organism is sensitive to the agents. According to Cushnie and Lamb [16], incubation at any temperatures other than the optimum often leads to slower growth of the organisms with consequent larger zone. It is clear that whatever temperature chosen for incubation, uniformity of the heating rate of the plates is more important than the absolute value of the incubation temperature. The depth of the medium in the Petri dish should also be of consistent thickness (about $3-4 \mathrm{~mm}$ ) in order to obtain a standard result because the inhibition zone will increase as the thickness of the agar decreases, and the effect is more marked with very thin plate. Among the main sources of error often occur in susceptibility test is the concentration of target organism inoculum [26]. Heavy inoculum tends to give false small inhibition zone, while light inoculum results in false large zone [27]. Therefore, broth dilution method was also carried out in the current study to confirm the results of the disc diffusion assay whereby the MIC and MMC values were determined.

\section{Conclusion}

This study shows that the crude flower and fruit extracts of M. malabathricum Linn. exhibit interesting antimicrobial properties. This may partly explain and support the use of various parts of this plant in traditional medicine for the treatment of infectious diseases caused by microorganisms. However, further studies in determining the bioactive compounds composition, pharmacological action and toxicity of these extracts should be addressed to confirm the therapeutic benefits of the plant prior future applications.

\section{Acknowledgements}

This research project was supported by the Ministry of Science, Technology and Innovation, Malaysia under the Grant 05-01-04-SF0231.

\section{REFERENCES}

[1] World Health Organization (WHO), "National Policy on Traditional Medicine and Regulation of Herbal Medicines,” Geneva, 2005.

[2] B. Bhattacharyya and B. M. Johri, "Flowering Plants: Taxonomy and Phylogeny,” Nurosa Publishing House, New Delhi, 1998.

[3] D. Susanti, M. S. Hasnah, A. Farediah, M. A. Rasadah, A. Norio and K. Mariko, "Antioxidant and Cytotoxicity Flavonoids from the Flowers of Melastoma malabathricum L.,” Food Chemistry, Vol. 103, No. 3, 2007, pp. 710-716. doi:10.1016/j.foodchem.2006.09.011

[4] J. B. Lowry, "Anthocyanins of the Melastomataceae, Myrtaceae and Some Allied Families,” Phytochemistry, Vol. 15, No. 4, 1976, pp. 513-516. doi:10.1016/S0031-9422(00)88960-8

[5] S. M. Jofrry, N. J. Yob, M. S. Rofiee, M. M. R. Meor Mohd. Affandi, Z. Suhaili, F. Othman, M. A. Abdah, M. N. M. Desa and Z. A. Zakaria, "Melastoma malabathricum (L.) Smith Ethnomedicinal Uses, Chemical Constituents, and Pharmacological Properties: A Review,” EvidenceBased Complementary and Alternative Medicine, Vol. 2012, 2011, p. 48.

[6] F. Lohézic-Le Dévéhat, A. Bakhtiar, C. Bézivin, M. Amoros and J. Boustie, "Antiviral and Cytotoxic of Some Indonesian Plants,” Fitoterapia, Vol. 73, No. 5, 2002, pp. 400-405. doi:10.1016/S0367-326X(02)00125-9

[7] Z. A. Zakaria, R. N. R. Mohd. Nor, G. H. Kumar, Z. D. F. Abdul Ghani, M. R. Sulaiman, G. R. Devi, A. M. Mat Jais, M. N. Somchit and C. A. Fatimah, "Antinociceptive, Anti-Inflammatory and Antipyretic Properties of Melastoma malabathricum Leaves Aqueous Extract in Experimental Animals," Canadian Journal of Physiology Pharmacology, Vol. 84, No. 12, 2006, pp. 1291-1299. doi:10.1139/y06-083

[8] F. Hussain, M. A. Abdulla, S. M. Noor, S. Ismail and H. M. Ali, "Gastroprotective Effects of Melastoma malabathricum Aqueous Leaf Extract Against Ethanol-Induced Gastric Ulser Iin Rats,” American Journal of Biochemistry and Biotechnology, Vol. 4, No. 4, 2008, pp. 438-441. doi:10.3844/ajbbsp.2008.438.441

[9] M. Zulaikah, I. Nazliana and I. B. Ahmad, "Penindasan Terpilih Gen-Gen Staphylococcus aureus Rintang Metisilin Yang Dirawat Dengan Ektrak Metanol Melastoma malabathricum," Sains Malaysiana, Vol. 37, No. 1, 2008, pp. 107-113.

[10] National Committee for Clinical Laboratory Standard (NCCLS), "Performance Standards for Antimicrobial Susceptibility Testing,” Twelfth International Supplement, M100-S12, Wayne, 2002.

[11] M. E. Arias, J. D. Gomez, N. M. Cudmani, M. A. Vattuone and M. I. Isla, “Antibacterial Activity of Ethanolic and Aqueous Extracts of Acacia aroma Gill. ex Hook et Arn,” Life Sciences, Vol. 75, No. 2, 2004, pp. 191-202. doi:10.1016/j.lfs.2003.12.007

[12] S. M. R. Rohani, M. Moradi, T. Mehdizadeh, S. S. SaeiDehkordi and M. W. Griffiths, "The Effect of Nisin and Garlic (Allium sativum L.) Essential Oil Separately and in 
Combination on the Growth of Listeria monocytogenes," LWT-Food Science and Technology, Vol. 44, 2011, pp. 2260-2265.

[13] B. B. Li, B. Smith and M. M. Hossain, "Extraction of Phenolics from Citrus Peels: I. Solvent Extraction Method," Separation and Purification Technology, Vol. 48, No. 2, 2006, pp. 182-188. doi:10.1016/j.seppur.2005.07.005

[14] V. Duraipandiyan and S. Ignacimuthu, "Antibacterial and Antifungal Activity of Cassia fistula L.: An Ethnomedicinal Plant,” Journal of Ethnopharmacology, Vol. 112, No. 3, 2007, pp. 590-594. doi:10.1016/j.jep.2007.04.008

[15] L. J. Shai, L. J. McGaw, P. Masoko and J. N. Eloff, “Antifungal and Antibacterial Activity of Seven Traditionally Used South African Plant Species Active against Candida albicans," South African Journal of Botany, Vol. 74, No. 4, 2008, pp. 677-684. doi:10.1016/j.sajb.2008.04.003

[16] T. P. T. Cushnie and A. J. Lamb, “Antimicrobial Activity of Flavonoids," International Journal of Antimicrobial Agents, Vol. 26, No. 5, 2005, pp. 343-356. doi:10.1016/j.ijantimicag.2005.09.002

[17] Y.-C. Wang, H.-W. Hsu and W.-L. Liao, “Antibacterial Activity of Melastoma candidum D. Don," LWT_Food Science and Technology, Vol. 41, No. 10, 2008, pp. 17931798.

[18] H. N. Thatoi, S. K. Panda, S. K. Rath and S. K. Dutta, "Antimicrobial Activity and Ethnomedicinal Uses of Some Medicinal Plants from Similipal Biosphere Reserve, Orissa," Asian Journal of Plant Sciences, Vol. 7, No. 3, 2008, pp. 260-267. doi:10.3923/ajps.2008.260.267

[19] P. W. Grosvenor, A. Supriono and D. O. Gray, "Medicinal Plants from Riau Province, Sumatra, Indonesia. Part 2: Antibacterial and Antifungal Activity," Journal of Ethnopharmacology, Vol. 45, No. 2, 1995, pp. 97-111. doi:10.1016/0378-8741(94)01210-Q

[20] B. Shan, Y.-Z. Cai, J. D. Brooks and H. Corke, "The in Vitro Antibacterial Activity of Dietary Spice and Medical
Herbs Extracts,” International Journal of Food Microbiology, Vol. 117, No. 1, 2007, pp. 112-119. doi:10.1016/j.ijfoodmicro.2007.03.003

[21] E. Cheylan and D. Y. C. Fung, "Antimicrobial Activity of Spices," Journal of Rapid Method and Automation in Microbiology, Vol. 12, No. 1, 2004, pp. 1-55. doi:10.1111/j.1745-4581.2004.tb00046.X

[22] P. Lopez, C. Sanchez, R. Batlle and C. Nerin, "Solid- and Vapor-Phase Antimicrobial Activities of Six Essential Oils: Susceptibility of Selected Foodborne Bacteria and Fungal Strains," Journal of Agriculture and Food Chemistry, Vol. 53, No. 17, 2005, pp. 6939-6946. doi:10.1021/jf050709v

[23] C. F. Duffy and R. F. Power, "Antioxidant and Antimicrobial Properties of Some Chinese Plant Extracts," International Journal of Antimicrobial Agents, Vol. 17, No. 6, 2001, pp. 527-529.

[24] Y. Gao, M. J. Van Belkum and M. E. Stiles, "The Outer Membrane of Gram-Negative Bacteria Inhibits Antibacterial Activity of Brochocin-C," Applied and Environmental Microbiology, Vol. 65, No. 10, 1999, pp. 43294333.

[25] J. Côté, S. Caillet, G. Doyon, D. Dussault, J.-F. Sylvain and M. Lacroix, "Antimicrobial Effect of Cranberry Juice and Extracts," Food Control, Vol. 22, No. 8, 2011, pp. 1413-1418. doi:10.1016/j.foodcont.2011.02.024

[26] T. Taguri, T. Tanaka and I. Kouno, “Antimicrobial Activity of 10 Different Plant Polyphenols against Bacteria Causing Food-Borne Disease,” Biological and Pharmaceutical Bulletin, Vol. 27, No. 12, 2004, pp. 1965-1969. doi:10.1248/bpb.27.1965

[27] A. Basile, S. Sorbo, S. Giordano, L. Ricciardi, S. Ferrara, D. Montesano, R. C. Cobianchi, M. L. Vuotto and L. Ferrara, "Antibaterial and Allelopathic Activity of Extract from Castanea sativa Leaves,” Fitoterapia, Vol. 71, No. 1, 2000, pp. S110-S116. doi:10.1016/S0367-326X(00)00185-4 